\title{
FOUR DIMENSIONAL BIHARMONIC HYPERSURFACES IN NONZERO SPACE FORM HAVE CONSTANT MEAN CURVATURE
}

\author{
ZHIDA GUAN, HAIZHONG LI, AND LUC VRANCKEN
}

\begin{abstract}
In this paper, through making careful analysis of Gauss and Codazzi equations, we prove that four dimensional biharmonic hypersurfaces in nonzero space form have constant mean curvature. Our result gives the positive answer to the conjecture proposed by Balmus-Montaldo-Oniciuc in 2008 for four dimensional hypersurfaces.
\end{abstract}

\section{INTRODUCTION}

Biharmonic maps were introduced in 1964 by Eells and Sampson [ES] as a generalization of harmonic maps. In their paper, Eells and Sampson suggested considering the bi-energy of a map $\phi:\left(M^{n}, g\right) \rightarrow\left(N^{m}, h\right)$ between two Riemannian manifolds defined by

$$
E_{2}(\phi)=\frac{1}{2} \int_{M^{n}}|\tau(\phi)|^{2} d \mu_{g}
$$

where $\tau(\phi)$ is the tension field of $\phi$ and $d \mu_{g}$ is the volume element on $\left(M^{n}, g\right)$. Stationary points of the bi-energy functional are called biharmonic maps. Jiang (see [J1], [J2]) is the first mathematician who systematically studied the bi-energy functional, and he computed the first and second variations of $E_{2}$. The stationary points of the functional $E_{2}$ satisfy the Euler-Lagrange equation

$$
-\Delta \tau(\phi)=\sum_{i=1}^{n} R^{N^{m}}\left(d \phi\left(e_{i}\right), \tau(\phi)\right) d \phi\left(e_{i}\right),
$$

where $\Delta$ is the Laplacian of $\left(M^{n}, g\right)$. Biharmonic submanifolds have attracted a lot of attentions from mathematicians and many important results on biharmonic submanifolds have been obtained since then (see [C1], [C2], [BMO1], [BMO2], [CMO1], [CMO2], [F1], [F2], [F3], [FH]).

The following conjecture was proposed by Balmus-Montaldo-Oniciuc in 2008 [BMO1] (also see Conjecture 7.2 of page 180 in [OC]).

Conjecture. Any $n$-dimensional biharmonic submanifold in $\mathbb{S}^{n+p}$ has constant mean curvature.

When $n=2, p=1$, the conjecture was proved by Caddeo-Montaldo-Oniciuc in [CMO1]; when $n=3, p=1$, the conjecture was proved by Balmus-MontaldoOniciuc in [BMO2]. In this paper, we prove the conjecture for $n=4, p=1$. In fact, we prove the following theorem:

2010 Mathematics Subject Classification. Primary 53C40, 58E20; Secondary 53C42.

Key words and phrases. Biharmonic maps, Biharmonic hypersurfaces, Constant mean curvature. 
Theorem 1.1. Four dimensional biharmonic hypersurfaces in nonzero space form $\mathbb{R}^{5}(c)(c \neq 0)$ have constant mean curvature.

In the study of biharmonic submanifolds, there are two other conjectures proposed by Chen in 1991 [C1], and by Caddeo-Montaldo-Oniciuc in 2001 [CMO1], respectively.

Chen's Conjecture. Every n-dimensional biharmonic submanifold of Euclidean spaces $\mathbb{R}^{n+p}$ is minimal.

Generalized Chen's Conjecture. Every n-dimensional biharmonic submanifold of a Riemannian manifold $N^{n+p}$ with non-positive sectional curvature is minimal.

When $n=2, p=1$, Chen's conjecture was proved by Chen and Jiang around 1987 independently; when $n=3, p=1$, Chen's conjecture was proved by Hasanis and Vlachos in 1995 [HV]. Recently, Fu-Hong-Zhan [FHZ] have made important progress about Chen's conjecture. In fact, they proved Chen's conjecture for $n=$ $4, p=1$.

$\mathrm{Ou}$ and Tang $[\mathrm{OT}]$ constructed a family of counterexamples, where the generalized Chen's conjecture is false when the ambient space has nonconstant negative sectional curvature. However, the generalized Chen's conjecture remains open when the ambient spaces have constant sectional curvature. In particular, when $p=1, N^{n+1}=\mathbb{H}^{n+1}, n=2$ and $n=3$, the generalized Chen's conjecture was proved by Caddeo-Montaldo-Oniciuc [CMO2] and Balmus-Montaldo-Oniciuc [BMO2], respectively. When $c=-1$, our Theorem 1.1 solves the generalized Chen's conjecture for $p=1, N^{n+1}=\mathbb{H}^{n+1}, n=4$.

The paper is organized as follows. In Section 2, we recall some fundamental concepts and formulas for $n$-dimensional biharmonic hypersurfaces in space forms $\mathbb{R}^{n+1}(c)$. In Section 3, for 4-dimensional biharmonic hypersurfaces, we derive some equations and lemmas. In Section 4, we give the proof of Theorem 1.1.

Acknowledgement: The authors are supported by NSFC-FWO grant No.11961131001. The first two authors are supported by NSFC grant No. 11831005 and No. 11671224 .

\section{Preliminaries}

Let $M^{n}$ be an $n$-dimensional hypersurface in $(n+1)$-dimensional space form $\mathbb{R}^{n+1}(c)$ with constant sectional curvature $c$. When $c=0, \mathbb{R}^{n+1}(c)$ is $(n+1)$ dimensional Euclidean space; when $c=1, \mathbb{R}^{n+1}(c)$ is $(n+1)$-dimensional unit sphere; when $c=-1, \mathbb{R}^{n+1}(c)$ is $(n+1)$-dimensional hyperbolic space. Let $\nabla$ and $\tilde{\nabla}$ be the Levi-Civita connections of $M^{n}$ and $\mathbb{R}^{n+1}(c)$. Denote $X$ and $Y$ tangent vector fields of $M^{n}$ and $\xi$ the unit normal vector field. Then the Gauss formula and Weingarten formula (for example, see [C3]) are

$$
\begin{gathered}
\tilde{\nabla}_{X} Y=\nabla_{X} Y+h(X, Y) \xi, \\
\tilde{\nabla}_{X} \xi=-A X,
\end{gathered}
$$

where $h$ is the second fundamental form, and $A$ is the Weingarten operator. The mean curvature function $H$ is defined by 


$$
H=\frac{1}{n} \text { trace } h
$$

Moreover, the Gauss and Codazzi equations are given by

$$
\begin{gathered}
R(X, Y) Z=c(\langle Y, Z\rangle X-\langle X, Z\rangle Y)+\langle A Y, Z\rangle A X-\langle A X, Z\rangle A Y, \\
\left(\nabla_{X} A\right) Y=\left(\nabla_{Y} A\right) X,
\end{gathered}
$$

where the Riemannian curvature $R(X, Y) Z$ is defined by

$$
R(X, Y) Z=\nabla_{X} \nabla_{Y} Z-\nabla_{Y} \nabla_{X} Z-\nabla_{[X, Y]} Z .
$$

We have the following characterization result for $M^{n}$ to be a biharmonic hypersurface in $\mathbb{R}^{n+1}(c)$ (see [J1], [J2], [BMO2], [CMO2], [F3], [FH]).

Proposition 2.1. A hypersurface $M^{n}$ in a space form $\mathbb{R}^{n+1}(c)$ is biharmonic if and only if $H$ and $A$ satisfy

$$
\begin{gathered}
\Delta H+H \text { trace } A^{2}=n c H, \\
2 A \operatorname{grad} H+n H \operatorname{grad} H=0,
\end{gathered}
$$

where the Laplacian operator $\Delta$ acting on a smooth function $f$ on $M^{n}$ is defined by

$$
\Delta f=-\operatorname{div}(\nabla f) .
$$

Let $M^{n}$ be an $n$-dimensional biharmonic hypersurface in $(n+1)$-dimensional space form $\mathbb{R}^{n+1}(c)$. Suppose the mean curvature function $H$ is not constant. From (2.8), we have that grad $H$ is an eigenvector of the Weingarten operator $A$ with the corresponding principal curvature $-n H / 2$. Without loss of generality, we can choose $e_{1}$ such that $e_{1}$ is parallel to $\operatorname{grad} H$, and we can choose suitable orthonormal frame $\left\{e_{1}, e_{2}, \cdots, e_{n}\right\}$ such that

$$
A e_{i}=\lambda_{i} e_{i}
$$

where $\lambda_{1}=-n H / 2$.

Denote the connection coefficients $\omega_{i j}^{k}$ by

$$
\nabla_{e_{i}} e_{j}=\sum_{k=1}^{n} \omega_{i j}^{k} e_{k}, \quad \omega_{i j}^{k}+\omega_{i k}^{j}=0, \quad i, j=1, \cdots, n .
$$

Lemma 2.2. ([FH]) Let $M^{n}$ be an biharmonic hypersurface in a space form $\mathbb{R}^{n+1}(c)$ and assume the mean curvature $H$ is non-constant. Then the multiplicity of the principal curvature $\lambda_{1}(=-n H / 2)$ is one, that is, $\lambda_{j} \neq \lambda_{1}$ for $2 \leq j \leq n$. 
Lemma 2.3. ([FH]) Let $M^{n}$ be an biharmonic hypersurface in a space form $\mathbb{R}^{n+1}(c)$ and assume the mean curvature $H$ is non-constant. Then the principal curvatures $\lambda_{i}$ and the connection coefficients $\omega_{i i}^{1}$ satisfy

$$
\begin{gathered}
e_{1} e_{1}\left(\lambda_{1}\right)=e_{1}\left(\lambda_{1}\right)\left(\sum_{j=2}^{n} \omega_{j j}^{1}\right)+\lambda_{1}\left(n(n-2) c-R+4 \lambda_{1}^{2}\right), \\
e_{1}\left(\lambda_{i}\right)=\left(\lambda_{i}-\lambda_{1}\right) \omega_{i i}^{1}, \quad 2 \leq i \leq n \\
e_{1}\left(\omega_{i i}^{1}\right)=\left(\omega_{i i}^{1}\right)^{2}+\lambda_{1} \lambda_{i}+c, \quad 2 \leq i \leq n,
\end{gathered}
$$

where $R$ is the scalar curvature.

In 2015, Fu [F2] proved the following theorem.

Theorem 2.4. ([F2]) Let $M^{n}$ be a biharmonic hypersurface with at most three distinct principal curvatures in $\mathbb{R}^{n+1}(c)$. Then $M^{n}$ has constant mean curvature.

\section{Four Dimensional BIHARMONIC HYPERSURFACES IN $\mathbb{R}^{5}(c)$}

From now on, we study the biharmonicity of a hypersurface $M^{4}$ in a space form $\mathbb{R}^{5}(c)$. By Theorem 2.4, we only need to work on the case that $M^{4}$ has four distinct principal curvatures, and we assume that the mean curvature $H$ is non-constant. Then there exists a neighborhood of $p$ such that $\operatorname{grad} H \neq 0$. The squared length of the second fundamental form of $M$ is

$$
S=\sum_{i=1}^{4} \lambda_{i}^{2}=4 H^{2}+\lambda_{2}^{2}+\lambda_{3}^{2}+\lambda_{4}^{2} .
$$

By using $\lambda_{1}=-2 H$, Gauss equation is

$$
R=12 c+16 H^{2}-S=12 c+12 H^{2}-\lambda_{2}^{2}-\lambda_{3}^{2}-\lambda_{4}^{2} .
$$

Since $e_{1}$ is parallel to $\operatorname{grad} H$, it follows that

$$
e_{1}(H) \neq 0, \quad e_{2}(H)=e_{3}(H)=e_{4}(H)=0 .
$$

The following result can be found in [F3].

Lemma 3.1. ([F3]) Let $M^{4}$ be a biharmonic hypersurface with four distinct principal curvatures in space forms $\mathbb{R}^{5}(c)$, then we have

$$
\begin{gathered}
\nabla_{e_{1}} e_{i}=0, \quad i=1,2,3,4, \\
\nabla_{e_{i}} e_{1}=-\omega_{i i}^{1} e_{i}, \quad i=2,3,4, \\
\nabla_{e_{i}} e_{i}=\sum_{k=1, k \neq i}^{4} \omega_{i i}^{k} e_{k}, \quad i=2,3,4, \\
\nabla_{e_{i}} e_{j}=-\omega_{i i}^{j} e_{i}+\omega_{i j}^{k} e_{k} \quad \text { for distinct } i, j, k=2,3,4,
\end{gathered}
$$

where

$$
\omega_{i i}^{j}=-\frac{e_{j}\left(\lambda_{i}\right)}{\lambda_{j}-\lambda_{i}}
$$


Note that we have

$$
\begin{gathered}
\lambda_{2}+\lambda_{3}+\lambda_{4}=-3 \lambda_{1}, \\
S=\sum_{i=1}^{4} \lambda_{i}^{2}=\lambda_{1}^{2}+\sum_{i=2}^{4} \lambda_{i}^{2}, \\
e_{1}\left(\lambda_{1}\right) \neq 0, \quad e_{i}\left(\lambda_{1}\right)=0, \quad 2 \leq i \leq 4 .
\end{gathered}
$$

Following $[\mathrm{FH}]$ and $[\mathrm{FHZ}]$, the functions $f_{k}$ are defined by

$$
f_{k}=\left(\omega_{22}^{1}\right)^{k}+\left(\omega_{33}^{1}\right)^{k}+\left(\omega_{44}^{1}\right)^{k}, \quad k=1, \cdots, 5 .
$$

The following lemma was proved by Fu-Hong-Zhan in [FHZ].

Lemma 3.2. ([FHZ]) With the notations $f_{k}$, the following two relations hold

$$
\begin{gathered}
f_{1}^{4}-6 f_{1}^{2} f_{2}+3 f_{2}^{2}+8 f_{1} f_{3}-6 f_{4}=0 \\
f_{1}^{5}-5 f_{1}^{3} f_{2}+5 f_{1}^{2} f_{3}+5 f_{2} f_{3}-6 f_{5}=0 .
\end{gathered}
$$

For simplicity, given a function $g$ on $M^{4}$, we write $g^{\prime}=e_{1}(g), g^{\prime \prime}=e_{1} e_{1}(g), g^{\prime \prime \prime}=$ $e_{1} e_{1} e_{1}(g)$ and $g^{\prime \prime \prime \prime}=e_{1} e_{1} e_{1} e_{1}(g)$. Also, we write $\lambda=\lambda_{1}$ and $f_{1}=T$. Note that for $i=2,3,4$, using Lemma 3.1, we have

$$
\begin{aligned}
e_{i}\left(\lambda^{\prime}\right) & =e_{i} e_{1}(\lambda) \\
& =e_{1} e_{i}(\lambda)+\left[e_{i}, e_{1}\right](\lambda) \\
& =\left(\nabla_{e_{i}} e_{1}-\nabla_{e_{1}} e_{i}\right)(\lambda)=0,
\end{aligned}
$$

similarly we have

$$
e_{i}\left(\lambda^{\prime \prime}\right)=e_{i}\left(\lambda^{\prime \prime \prime}\right)=e_{i}\left(\lambda^{\prime \prime \prime \prime}\right)=e_{i}\left(\lambda^{\prime \prime \prime \prime \prime \prime}\right)=0 .
$$

Following the argument of Fu-Hong-Zhan in [FHZ], we can prove

Lemma 3.3. $f_{k}$ can be written as

$$
\left\{\begin{aligned}
f_{1}= & T \\
f_{2}= & T^{\prime}+3 \lambda^{2}-3 c \\
f_{3}= & \frac{1}{2} T^{\prime \prime}-\left(\lambda^{2}+c\right) T+6 \lambda \lambda^{\prime}, \\
f_{4}= & \frac{1}{6} T^{\prime \prime \prime}-\frac{4}{3}\left(\lambda^{2}+c\right) T^{\prime}-\frac{5}{3} \lambda \lambda^{\prime} T+2 \lambda^{\prime 2}+4 \lambda \lambda^{\prime \prime}-2 \lambda^{4}-c \lambda^{2}+3 c^{2}, \\
f_{5}= & \frac{1}{24} T^{\prime \prime \prime \prime}-\frac{5}{6}\left(\lambda^{2}+c\right) T^{\prime \prime}+2 \lambda \lambda^{\prime \prime \prime}+\frac{5}{3} \lambda^{\prime} \lambda^{\prime \prime}-\frac{26}{3} \lambda^{3} \lambda^{\prime}-\frac{47}{6} c \lambda \lambda^{\prime} \\
& -\frac{25}{12} \lambda \lambda^{\prime} T^{\prime}-\frac{1}{12}\left(13 \lambda \lambda^{\prime \prime}+\lambda^{\prime 2}-12 \lambda^{4}-24 c \lambda^{2}-12 c^{2}\right) T .
\end{aligned}\right.
$$


Proof. Since $e_{1}(\lambda) \neq 0$, from (2.12), we have

$$
f_{1}=\frac{e_{1} e_{1}(\lambda)-\lambda(S-4 c)}{e_{1}(\lambda)}=\frac{\lambda^{\prime \prime}}{\lambda^{\prime}}-\frac{\lambda}{\lambda^{\prime}}(S-4 c)=: T .
$$

Taking the sum of $i$ from 2 to 4 in (2.14) and (2.13) and using (3.9), we have

$$
\begin{aligned}
f_{2}=3 \lambda^{2} & +e_{1}\left(f_{1}\right)-3 c=T^{\prime}+3 \lambda^{2}-3 c, \\
g_{1}: & =\sum_{i=2}^{4} \lambda_{i} \omega_{i i}^{1} \\
& =\lambda T-3 e_{1}(\lambda)=\lambda T-3 \lambda^{\prime} .
\end{aligned}
$$

Multiplying $\omega_{i i}^{1}$ on both sides of (2.14), we have

$$
\frac{1}{2} e_{1}\left(\left(\omega_{i i}^{1}\right)^{2}\right)=\left(\omega_{i i}^{1}\right)^{3}+\lambda \lambda_{i} \omega_{i i}^{1}+c \omega_{i i}^{1} .
$$

Taking the sum of $i$ from 2 to 4 , we have

$$
\begin{aligned}
f_{3} & =\frac{1}{2} e_{1}\left(f_{2}\right)-\lambda g_{1}-c T \\
& =\frac{1}{2} T^{\prime \prime}-\left(\lambda^{2}+c\right) T+6 \lambda \lambda^{\prime} .
\end{aligned}
$$

Differentiating (3.20) with respect to $e_{1}$ and using (2.13) and (2.14), we have

$$
e_{1}\left(g_{1}\right)=2 \sum_{i=2}^{4} \lambda_{i}\left(\omega_{i i}^{1}\right)^{2}+\lambda \sum_{i=2}^{4} \lambda_{i}^{2}-\lambda \sum_{i=2}^{4}\left(\omega_{i i}^{1}\right)^{2}-3 c \lambda .
$$

From (3.9), (3.10), (3.18), we have

$$
\begin{aligned}
g_{2}:=\sum_{i=2}^{4} \lambda_{i}\left(\omega_{i i}^{1}\right)^{2} & =\frac{1}{2}\left\{e_{1}\left(g_{1}\right)-\lambda\left(S-\lambda^{2}\right)+\lambda f_{2}+3 c \lambda\right\} \\
& =\frac{1}{2}\left\{e_{1}\left(g_{1}\right)-\lambda^{\prime \prime}+\lambda^{\prime} T+\lambda^{3}+\lambda f_{2}-c \lambda\right\} .
\end{aligned}
$$

Using (3.19) and (3.20), we have

$$
g_{2}=\lambda T^{\prime}+\lambda^{\prime} T-2 \lambda^{\prime \prime}+2 \lambda^{3}-2 c \lambda .
$$

Multiplying $\left(\omega_{i i}^{1}\right)^{2}$ on both sides of $(2.14)$, we have

$$
\frac{1}{3} e_{1}\left(\left(\omega_{i i}^{1}\right)^{3}\right)=\left(\omega_{i i}^{1}\right)^{4}+\lambda \lambda_{i}\left(\omega_{i i}^{1}\right)^{2}+c\left(\omega_{i i}^{1}\right)^{2} .
$$

Taking the sum of $i$ from 2 to 4 , we have

$$
\begin{aligned}
f_{4} & =\frac{1}{3} e_{1}\left(f_{3}\right)-\lambda g_{2}-c f_{2} \\
& =\frac{1}{6} T^{\prime \prime \prime}-\frac{4}{3}\left(\lambda^{2}+c\right) T^{\prime}-\frac{5}{3} \lambda \lambda^{\prime} T+2 \lambda^{\prime 2}+4 \lambda \lambda^{\prime \prime}-2 \lambda^{4}-c \lambda^{2}+3 c^{2} .
\end{aligned}
$$

Multiplying $\lambda_{i}$ on both sides of (2.13), we have

$$
\lambda_{i}^{2} \omega_{i i}^{1}=\frac{1}{2} e_{1}\left(\lambda_{i}^{2}\right)+\lambda \lambda_{i} \omega_{i i}^{1} .
$$


Using (3.10), we have

$$
\begin{aligned}
g_{3}:=\sum_{i=2}^{4} \lambda_{i}^{2} \omega_{i i}^{1} & =\frac{1}{2} e_{1}\left(S-\lambda^{2}\right)+\lambda g_{1} \\
& =\frac{1}{2}\left(\frac{\lambda^{\prime \prime}-\lambda^{\prime} T}{\lambda}-\lambda^{2}\right)^{\prime}+\lambda g_{1} \\
& =-\frac{\lambda^{\prime}}{2 \lambda} T^{\prime}+\left(\lambda^{2}-\frac{\lambda^{\prime \prime} \lambda-\lambda^{\prime 2}}{2 \lambda^{2}}\right) T-4 \lambda \lambda^{\prime}+\frac{\lambda^{\prime \prime \prime} \lambda-\lambda^{\prime \prime} \lambda^{\prime}}{2 \lambda^{2}} .
\end{aligned}
$$

Differentiating (3.23) with respect to $e_{1}$ and using (2.13) and (2.14), we have

$$
e_{1}\left(g_{2}\right)=3 \sum_{i=2}^{4} \lambda_{i}\left(\omega_{i i}^{1}\right)^{3}-\lambda \sum_{i=2}^{4}\left(\omega_{i i}^{1}\right)^{3}+2 \lambda \sum_{i=2}^{4} \lambda_{i}^{2} \omega_{i i}^{1}+2 c \sum_{i=2}^{4} \lambda_{i} \omega_{i i}^{1},
$$

that is

$$
\begin{aligned}
g_{4}:= & \sum_{i=2}^{4} \lambda_{i}\left(\omega_{i i}^{1}\right)^{3} \\
= & \frac{1}{3}\left(e_{1}\left(g_{2}\right)+\lambda f_{3}-2 \lambda g_{3}-2 c g_{1}\right) \\
= & \frac{1}{2} \lambda T^{\prime \prime}+\lambda^{\prime} T^{\prime}+\frac{1}{3}\left(2 \lambda^{\prime \prime}-3 \lambda^{3}-\frac{\lambda^{\prime 2}}{\lambda}-3 c \lambda\right) T \\
& -\lambda^{\prime \prime \prime}+\frac{20}{3} \lambda^{2} \lambda^{\prime}+\frac{\lambda^{\prime \prime} \lambda^{\prime}}{3 \lambda}+\frac{4}{3} c \lambda^{\prime} .
\end{aligned}
$$

Multiplying $\left(\omega_{i i}^{1}\right)^{3}$ on both sides of (2.14), we have

$$
\frac{1}{4} e_{1}\left(\left(\omega_{i i}^{1}\right)^{4}\right)=\left(\omega_{i i}^{1}\right)^{5}+\lambda \lambda_{i}\left(\omega_{i i}^{1}\right)^{3}+c\left(\omega_{i i}^{1}\right)^{3} .
$$

Taking the sum of $i$ from 2 to 4 , we have

$$
\begin{aligned}
f_{5}= & \frac{1}{4} e_{1}\left(f_{4}\right)-\lambda g_{4}-c f_{3} \\
= & \frac{1}{24} T^{\prime \prime \prime \prime}-\frac{5}{6}\left(\lambda^{2}+c\right) T^{\prime \prime}+2 \lambda \lambda^{\prime \prime \prime}+\frac{5}{3} \lambda^{\prime} \lambda^{\prime \prime}-\frac{26}{3} \lambda^{3} \lambda^{\prime}-\frac{47}{6} c \lambda \lambda^{\prime} \\
& -\frac{25}{12} \lambda \lambda^{\prime} T^{\prime}-\frac{1}{12}\left(13 \lambda \lambda^{\prime \prime}+\lambda^{\prime 2}-12 \lambda^{4}-24 c \lambda^{2}-12 c^{2}\right) T .
\end{aligned}
$$

Following the argument of Fu-Hong-Zhan in [FHZ], we can prove

Lemma 3.4. The function $T$ satisfies $e_{i}(T)=0$ for $i=2,3,4$.

Proof. Assume that $T \neq 0$. Substituting (3.17) into (3.13) and (3.14), we have

$$
\begin{aligned}
& 9 c^{2}+10 c T^{2}+T^{4}-48 c \lambda^{2}-26 T^{2} \lambda^{2}+39 \lambda^{4} \\
- & 10 c T^{\prime}-6 T^{2} T^{\prime}+26 \lambda^{2} T^{\prime}+3 T^{2}+58 T \lambda \lambda^{\prime}-12 \lambda^{\prime 2} \\
+ & 4 T T^{\prime \prime}-24 \lambda \lambda^{\prime \prime}-T^{\prime \prime \prime}=0,
\end{aligned}
$$




$$
\begin{aligned}
& 36 c^{2} T+40 c T^{3}+4 T^{5}-48 c T \lambda^{2}-80 T^{3} \lambda^{2}-84 T \lambda^{4} \\
- & 20 c T T^{\prime}-20 T^{3} T^{\prime}-20 T \lambda^{2} T^{\prime}-172 c \lambda \lambda^{\prime}+120 T^{2} \lambda \lambda^{\prime}+568 \lambda^{3} \lambda^{\prime} \\
+ & 170 \lambda T^{\prime} \lambda^{\prime}+2 T \lambda^{\prime 2}-10 c T^{\prime \prime}+10 T^{2} T^{\prime \prime}+50 \lambda^{2} T^{\prime \prime}+10 T^{\prime} T^{\prime \prime} \\
+ & 26 T \lambda \lambda^{\prime \prime}-40 \lambda^{\prime} \lambda^{\prime \prime}-48 \lambda \lambda^{\prime \prime \prime}-T^{\prime \prime \prime \prime}=0 .
\end{aligned}
$$

Differentiating (3.28) with respect to $e_{1}$, we have

$$
\begin{aligned}
& 20 c T T^{\prime}+4 T^{3} T^{\prime}-52 T \lambda^{2} T^{\prime}-12 T T^{2}-96 c \lambda \lambda^{\prime}-52 T^{2} \lambda \lambda^{\prime} \\
+ & 156 \lambda^{3} \lambda^{\prime}+110 \lambda T^{\prime} \lambda^{\prime}+58 T \lambda^{\prime 2}-10 c T^{\prime \prime}-6 T^{2} T^{\prime \prime}+26 \lambda^{2} T^{\prime \prime} \\
+ & 10 T^{\prime} T^{\prime \prime}+58 T \lambda \lambda^{\prime \prime}-48 \lambda^{\prime} \lambda^{\prime \prime}+4 T T^{\prime \prime \prime}-24 \lambda \lambda^{\prime \prime \prime}-T^{\prime \prime \prime \prime}=0 .
\end{aligned}
$$

From (3.29) and (3.30), eliminating $T^{\prime \prime \prime \prime}$ we have

$$
\begin{aligned}
& -36 c^{2} T-40 c T^{3}-4 T^{5}+48 c T \lambda^{2}+80 T^{3} \lambda^{2}+84 T \lambda^{4}+40 c T T^{\prime} \\
& +24 T^{3} T^{\prime}-32 T \lambda^{2} T^{\prime}-12 T T^{2}+76 c \lambda \lambda^{\prime}-172 T^{2} \lambda \lambda^{\prime}-412 \lambda^{3} \lambda^{\prime} \\
& -60 \lambda T^{\prime} \lambda^{\prime}+56 T \lambda^{\prime 2}-16 T^{2} T^{\prime \prime}-24 \lambda^{2} T^{\prime \prime}+32 T \lambda \lambda^{\prime \prime}-8 \lambda^{\prime} \lambda^{\prime \prime} \\
& +4 T T^{\prime \prime \prime}+24 \lambda \lambda^{\prime \prime \prime}=0 .
\end{aligned}
$$

From (3.28) and (3.31), eliminating $T^{\prime \prime \prime}$ we have

$$
\begin{aligned}
& -36 c T \lambda^{2}-6 T^{3} \lambda^{2}+60 T \lambda^{4}+18 T \lambda^{2} T^{\prime}+19 c \lambda \lambda^{\prime}+15 T^{2} \lambda \lambda^{\prime} \\
& -103 \lambda^{3} \lambda^{\prime}-15 \lambda T^{\prime} \lambda^{\prime}+2 T \lambda^{\prime 2}-6 \lambda^{2} T^{\prime \prime}-16 T \lambda \lambda^{\prime \prime}-2 \lambda^{\prime} \lambda^{\prime \prime}+6 \lambda \lambda^{\prime \prime \prime}=0 .
\end{aligned}
$$

Differentiating (3.32) with respect to $e_{1}$, we have

$$
\begin{aligned}
& -36 c \lambda^{2} T^{\prime}-18 T^{2} \lambda^{2} T^{\prime}+60 \lambda^{4} T^{\prime}+18 \lambda^{2} T^{2}-72 c T \lambda \lambda^{\prime}-12 T^{3} \lambda \lambda^{\prime} \\
& +240 T \lambda^{3} \lambda^{\prime}+66 T \lambda T^{\prime} \lambda^{\prime}+19 c \lambda^{\prime 2}+15 T^{2} \lambda^{\prime 2}-309 \lambda^{2} \lambda^{\prime 2}-13 T^{\prime} \lambda^{\prime 2} \\
& +18 T \lambda^{2} T^{\prime \prime}-27 \lambda \lambda^{\prime} T^{\prime \prime}+19 c \lambda \lambda^{\prime \prime}+15 T^{2} \lambda \lambda^{\prime \prime}-103 \lambda^{3} \lambda^{\prime \prime}-31 \lambda T^{\prime} \lambda^{\prime \prime} \\
& -12 T \lambda^{\prime} \lambda^{\prime \prime}-2 \lambda^{\prime \prime 2}-6 \lambda^{2} T^{\prime \prime \prime}-16 T \lambda \lambda^{\prime \prime \prime}+4 \lambda^{\prime} \lambda^{\prime \prime \prime}+6 \lambda \lambda^{\prime \prime \prime \prime}=0 .
\end{aligned}
$$

From (3.28) and (3.33), eliminating $T^{\prime \prime \prime}$ we have

$$
\begin{aligned}
& 54 c^{2} \lambda^{2}+60 c T^{2} \lambda^{2}+6 T^{4} \lambda^{2}-288 c \lambda^{4}-156 T^{2} \lambda^{4}+234 \lambda^{6} \\
- & 24 c \lambda^{2} T^{\prime}-18 T^{2} \lambda^{2} T^{\prime}+96 \lambda^{4} T^{\prime}+72 c T \lambda \lambda^{\prime}+12 T^{3} \lambda \lambda^{\prime}+108 T \lambda^{3} \lambda^{\prime} \\
- & 66 T \lambda T^{\prime} \lambda^{\prime}-19 c \lambda^{\prime 2}-15 T^{2} \lambda^{\prime 2}+237 \lambda^{2} \lambda^{\prime 2}+13 T^{\prime} \lambda^{\prime 2}+6 T \lambda^{2} T^{\prime \prime} \\
+ & 27 \lambda \lambda^{\prime} T^{\prime \prime}-19 c \lambda \lambda^{\prime \prime}-15 T^{2} \lambda \lambda^{\prime \prime}-41 \lambda^{3} \lambda^{\prime \prime}+31 \lambda T^{\prime} \lambda^{\prime \prime}+12 T \lambda^{\prime} \lambda^{\prime \prime} \\
+ & 2 \lambda^{\prime \prime 2}+16 T \lambda \lambda^{\prime \prime \prime}-4 \lambda^{\prime} \lambda^{\prime \prime \prime}-6 \lambda \lambda^{\prime \prime \prime \prime}=0 .
\end{aligned}
$$

From (3.32) and (3.34), eliminating $T^{\prime \prime}$ we have

$$
a_{1} T^{\prime}-a_{1} T^{2}+a_{2} T+a_{3}=0,
$$

where

$$
\begin{aligned}
a_{1}= & 62 \lambda^{2} \lambda^{\prime \prime}-109 \lambda \lambda^{\prime 2}+192 \lambda^{5}-48 c \lambda^{3}, \\
a_{2}= & 44 \lambda^{2} \lambda^{\prime \prime \prime}-124 \lambda \lambda^{\prime} \lambda^{\prime \prime}+550 \lambda^{4} \lambda^{\prime}+18 \lambda^{\prime 3}-142 c \lambda^{2} \lambda^{\prime}, \\
a_{3}= & -12 \lambda^{2} \lambda^{\prime \prime \prime \prime}+46 \lambda \lambda^{\prime} \lambda^{\prime \prime \prime}-82 \lambda^{4} \lambda^{\prime \prime}+4 \lambda \lambda^{\prime \prime 2} \\
& -18 \lambda^{\prime 2} \lambda^{\prime \prime}-453 \lambda^{3} \lambda^{\prime 2}+468 \lambda^{7}+108 c^{2} \lambda^{3}-576 c \lambda^{5}+133 c \lambda \lambda^{\prime 2}-38 c \lambda^{2} \lambda^{\prime \prime} .
\end{aligned}
$$

Case (i): $a_{1}=0, a_{2} \neq 0$. (3.35) becomes 


$$
a_{2} T+a_{3}=0 .
$$

so we have $T=-\frac{a_{3}}{a_{2}}$, then $e_{i}(T)=0$ for $i=2,3,4$.

Case (ii): $a_{1}=0, a_{2}=0$. We have

$$
\begin{gathered}
62 \lambda \lambda^{\prime \prime}-109 \lambda^{\prime 2}+192 \lambda^{4}-48 c \lambda^{2}=0, \\
44 \lambda^{2} \lambda^{\prime \prime \prime}-124 \lambda \lambda^{\prime} \lambda^{\prime \prime}+550 \lambda^{4} \lambda^{\prime}+18 \lambda^{\prime 3}-142 c \lambda^{2} \lambda^{\prime}=0 .
\end{gathered}
$$

Differentiating (3.36) with respect to $e_{1}$, from (3.37) we have

$$
-1145 c \lambda^{2}+77 \lambda^{4}+279 \lambda^{\prime 2}-206 \lambda \lambda^{\prime \prime}=0 .
$$

From (3.36) and (3.38), eliminating $\lambda^{\prime \prime}$ we have

$$
-40439 c \lambda^{2}+22163 \lambda^{4}-2578 \lambda^{\prime 2}=0 .
$$

Differentiating (3.39) with respect to $e_{1}$, we have

$$
-40439 c \lambda+44326 \lambda^{3}-2578 \lambda^{\prime \prime}=0 .
$$

Substituting (3.39) and (3.40) into (3.36), we have

$$
1776889 c+827421 \lambda^{2}=0,
$$

which is a contradiction.

Case (iii): $a_{1} \neq 0$. Differentiating (3.35) with respect to $e_{1}$, we have

$$
\begin{aligned}
& 96 c T \lambda^{3} T^{\prime}-384 T \lambda^{5} T^{\prime}+324 c^{2} \lambda^{2} \lambda^{\prime}+144 c T^{2} \lambda^{2} \lambda^{\prime}-2880 c \lambda^{4} \lambda^{\prime} \\
- & 960 T^{2} \lambda^{4} \lambda^{\prime}+3276 \lambda^{6} \lambda^{\prime}-286 c \lambda^{2} T^{\prime} \lambda^{\prime}+1510 \lambda^{4} T^{\prime} \lambda^{\prime}-284 c T \lambda \lambda^{\prime 2} \\
+ & 2200 T \lambda^{3} \lambda^{\prime 2}+218 T \lambda T^{\prime} \lambda^{\prime 2}+133 c \lambda^{\prime 3}+109 T^{2} \lambda^{\prime 3}-1359 \lambda^{2} \lambda^{\prime 3} \\
- & 91 T^{\prime} \lambda^{\prime 3}-48 c \lambda^{3} T^{\prime \prime}+192 \lambda^{5} T^{\prime \prime}-109 \lambda \lambda^{\prime 2} T^{\prime \prime}-142 c T \lambda^{2} \lambda^{\prime \prime} \\
+ & 550 T \lambda^{4} \lambda^{\prime \prime}-124 T \lambda^{2} T^{\prime} \lambda^{\prime \prime}+190 c \lambda \lambda^{\prime} \lambda^{\prime \prime}+94 T^{2} \lambda \lambda^{\prime} \lambda^{\prime \prime}-1234 \lambda^{3} \lambda^{\prime} \lambda^{\prime \prime} \\
- & 218 \lambda T^{\prime} \lambda^{\prime} \lambda^{\prime \prime}-70 T \lambda^{\prime 2} \lambda^{\prime \prime}+62 \lambda^{2} T^{\prime \prime} \lambda^{\prime \prime}-124 T \lambda \lambda^{\prime \prime 2}-32 \lambda^{\prime} \lambda^{\prime \prime 2} \\
- & 38 c \lambda^{2} \lambda^{\prime \prime \prime}-62 T^{2} \lambda^{2} \lambda^{\prime \prime \prime}-82 \lambda^{4} \lambda^{\prime \prime \prime}+106 \lambda^{2} T^{\prime} \lambda^{\prime \prime \prime}-36 T \lambda \lambda^{\prime} \lambda^{\prime \prime \prime} \\
+ & 28 \lambda^{\prime 2} \lambda^{\prime \prime \prime}+54 \lambda \lambda^{\prime \prime} \lambda^{\prime \prime \prime}+44 T \lambda^{2} \lambda^{\prime \prime \prime \prime}+22 \lambda \lambda^{\prime} \lambda^{\prime \prime \prime \prime}-12 \lambda^{2} \lambda^{\prime \prime \prime \prime \prime}=0 .
\end{aligned}
$$

From (3.32) and (3.41), eliminating $T^{\prime \prime}$ we have

$$
\begin{aligned}
& 1728 c^{2} T \lambda^{4}+288 c T^{3} \lambda^{4}-9792 c T \lambda^{6}-1152 T^{3} \lambda^{6} \\
+ & 11520 T \lambda^{8}-288 c T \lambda^{4} T^{\prime}+1152 T \lambda^{6} T^{\prime}+1032 c^{2} \lambda^{3} \lambda^{\prime} \\
+ & 144 c T^{2} \lambda^{3} \lambda^{\prime}-8688 c \lambda^{5} \lambda^{\prime}-2880 T^{2} \lambda^{5} \lambda^{\prime}-120 \lambda^{7} \lambda^{\prime} \\
- & 996 c \lambda^{3} T^{\prime} \lambda^{\prime}+6180 \lambda^{5} T^{\prime} \lambda^{\prime}+2124 c T \lambda^{2} \lambda^{\prime 2}+654 T^{3} \lambda^{2} \lambda^{\prime 2} \\
+ & 7044 T \lambda^{4} \lambda^{\prime 2}-654 T \lambda^{2} T^{\prime} \lambda^{\prime 2}-1273 c \lambda \lambda^{\prime 3}-981 T^{2} \lambda \lambda^{\prime 3} \\
+ & 3073 \lambda^{3} \lambda^{\prime 3}+1089 \lambda T^{\prime} \lambda^{\prime 3}-218 T \lambda^{\prime 4}-2316 c T \lambda^{3} \lambda^{\prime \prime} \\
- & 372 T^{3} \lambda^{3} \lambda^{\prime \prime}+3948 T \lambda^{5} \lambda^{\prime \prime}+372 T \lambda^{3} T^{\prime} \lambda^{\prime \prime}+2414 c \lambda^{2} \lambda^{\prime} \lambda^{\prime \prime} \\
+ & 1494 T^{2} \lambda^{2} \lambda^{\prime} \lambda^{\prime \prime}-14174 \lambda^{4} \lambda^{\prime} \lambda^{\prime \prime}-2238 \lambda^{2} T^{\prime} \lambda^{\prime} \lambda^{\prime \prime}+1448 T \lambda \lambda^{\prime 2} \lambda^{\prime \prime} \\
+ & 218 \lambda^{\prime 3} \lambda^{\prime \prime}-1736 T \lambda^{2} \lambda^{\prime \prime 2}-316 \lambda \lambda^{\prime} \lambda^{\prime \prime 2}-516 c \lambda^{3} \lambda^{\prime \prime \prime} \\
- & 372 T^{2} \lambda^{3} \lambda^{\prime \prime \prime}+660 \lambda^{5} \lambda^{\prime \prime \prime}+636 \lambda^{3} T^{\prime} \lambda^{\prime \prime \prime}-216 T \lambda^{2} \lambda^{\prime} \lambda^{\prime \prime \prime} \\
- & 486 \lambda \lambda^{\prime 2} \lambda^{\prime \prime \prime}+696 \lambda^{2} \lambda^{\prime \prime} \lambda^{\prime \prime \prime}+264 T \lambda^{3} \lambda^{\prime \prime \prime \prime}+132 \lambda^{2} \lambda^{\prime} \lambda^{\prime \prime \prime \prime}-72 \lambda^{3} \lambda^{\prime \prime \prime \prime \prime \prime}=0 .
\end{aligned}
$$


From (3.35) and (3.42), eliminating $T^{\prime}$ we have

$$
b_{1} T+b_{2}=0
$$

where

$$
\begin{aligned}
b_{1}= & -6480 c^{3} \lambda^{6}+63936 c^{2} \lambda^{8}-204336 c \lambda^{10}+209088 \lambda^{12} \\
& -40350 c^{2} \lambda^{4} \lambda^{\prime 2}+237750 c \lambda^{6} \lambda^{\prime 2}-309288 \lambda^{8} \lambda^{\prime 2}+4812 c \lambda^{2} \lambda^{4} \\
& -227013 \lambda^{4} \lambda^{\prime 4}+520 \lambda^{\prime 6}+20898 c^{2} \lambda^{5} \lambda^{\prime \prime}-125856 c \lambda^{7} \lambda^{\prime \prime} \\
& +174078 \lambda^{9} \lambda^{\prime \prime}-25773 c \lambda^{3} \lambda^{\prime 2} \lambda^{\prime \prime}+302157 \lambda^{5} \lambda^{\prime 2} \lambda^{\prime \prime}-975 \lambda \lambda^{\prime 4} \lambda^{\prime \prime} \\
& -5622 c \lambda^{4} \lambda^{\prime \prime 2}-7830 \lambda^{6} \lambda^{\prime \prime 2}+1350 \lambda^{2} \lambda^{\prime 2} \lambda^{\prime \prime 2}-13640 \lambda^{3} \lambda^{\prime \prime 3} \\
& +19719 c \lambda^{4} \lambda^{\prime} \lambda^{\prime \prime \prime}-89523 \lambda^{6} \lambda^{\prime} \lambda^{\prime \prime \prime}-717 \lambda^{2} \lambda^{\prime 3} \lambda^{\prime \prime \prime}+18354 \lambda^{3} \lambda^{\prime} \lambda^{\prime \prime} \lambda^{\prime \prime \prime} \\
& -3498 \lambda^{4} \lambda^{\prime \prime \prime \prime}-2016 c \lambda^{5} \lambda^{\prime \prime \prime \prime}+8064 \lambda^{7} \lambda^{\prime \prime \prime \prime}-4578 \lambda^{3} \lambda^{\prime 2} \lambda^{\prime \prime \prime \prime} \\
& +2604 \lambda^{4} \lambda^{\prime \prime} \lambda^{\prime \prime \prime \prime}, \\
b_{2}= & 7254 c^{3} \lambda^{5} \lambda^{\prime}-78246 c^{2} \lambda^{7} \lambda^{\prime}+295434 c \lambda^{9} \lambda^{\prime}-364410 \lambda^{11} \lambda^{\prime} \\
& -4566 c^{2} \lambda^{3} \lambda^{\prime 3}-11349 c \lambda^{5} \lambda^{\prime 3}+361623 \lambda^{7} \lambda^{\prime 3}-760 c \lambda \lambda^{\prime 5} \\
& +19795 \lambda^{3} \lambda^{\prime 5}+18996 c^{2} \lambda^{4} \lambda^{\prime} \lambda^{\prime \prime}-66342 c \lambda^{6} \lambda^{\prime} \lambda^{\prime \prime}-146838 \lambda^{8} \lambda^{\prime} \lambda^{\prime \prime} \\
& -3926 c \lambda^{2} \lambda^{\prime 3} \lambda^{\prime \prime}+120509 \lambda^{4} \lambda^{\prime 3} \lambda^{\prime \prime}-520 \lambda^{\prime 5} \lambda^{\prime \prime}+10472 c \lambda^{3} \lambda^{\prime} \lambda^{\prime \prime 2} \\
& -143462 \lambda^{5} \lambda^{\prime} \lambda^{\prime \prime 2}+415 \lambda \lambda^{\prime 3} \lambda^{\prime \prime 2}-1330 \lambda^{2} \lambda^{\prime} \lambda^{\prime \prime 3}-5490 c^{2} \lambda^{5} \lambda^{\prime \prime \prime} \\
& +29448 c \lambda^{7} \lambda^{\prime \prime \prime}-21366 \lambda^{9} \lambda^{\prime \prime \prime}+5100 c \lambda^{3} \lambda^{\prime 2} \lambda^{\prime \prime \prime}-20178 \lambda^{5} \lambda^{\prime 2} \lambda^{\prime \prime \prime} \\
& +360 \lambda \lambda^{\prime 4} \lambda^{\prime \prime \prime}-5154 c \lambda^{4} \lambda^{\prime \prime} \lambda^{\prime \prime \prime}+28338 \lambda^{6} \lambda^{\prime \prime} \lambda^{\prime \prime \prime}+1050 \lambda^{2} \lambda^{\prime 2} \lambda^{\prime \prime} \lambda^{\prime \prime \prime} \\
& +5076 \lambda^{3} \lambda^{\prime \prime 2} \lambda^{\prime \prime \prime}-3657 \lambda^{3} \lambda^{\prime} \lambda^{\prime \prime \prime 2}-2286 c \lambda^{4} \lambda^{\prime} \lambda^{\prime \prime \prime \prime}+12438 \lambda^{6} \lambda^{\prime} \lambda^{\prime \prime \prime \prime} \\
& -165 \lambda^{2} \lambda^{\prime 3} \lambda^{\prime \prime \prime \prime}-2334 \lambda^{3} \lambda^{\prime} \lambda^{\prime \prime} \lambda^{\prime \prime \prime \prime}+954 \lambda^{4} \lambda^{\prime \prime \prime} \lambda^{\prime \prime \prime \prime}+432 c \lambda^{5} \lambda^{\prime \prime \prime \prime \prime} \\
& -1728 \lambda^{7} \lambda^{\prime \prime \prime \prime \prime \prime}+981 \lambda^{3} \lambda^{\prime 2} \lambda^{\prime \prime \prime \prime \prime}-558 \lambda^{4} \lambda^{\prime \prime} \lambda^{\prime \prime \prime \prime \prime \prime} .
\end{aligned}
$$

If $b_{1} \neq 0$, then $T=-\frac{b_{2}}{b_{1}}$, and $e_{i}(T)=0$ for $i=2,3,4$. If $b_{1}=0$, then $b_{2}=0$. Using the similar technique in [FHZ] and (3.15), (3.16), we can eliminate $\lambda^{\prime \prime \prime \prime \prime}, \lambda^{\prime \prime \prime \prime}, \lambda^{\prime \prime \prime}, \lambda^{\prime \prime}, \lambda^{\prime}$ and get a nontrivial polynomial of $\lambda$ with constant coefficients. Thus $\lambda$ is a constant, which is a contradiction.

Following the argument of Fu-Hong-Zhan in [FHZ], we can get

Lemma 3.5. Suppose $M^{4}$ has four distinct principal curvatures, then $e_{j}\left(\lambda_{i}\right)=0$ for $2 \leq i, j \leq 4$.

Moreover, from (3.8) we have $\omega_{i i}^{j}=0$ for $2 \leq i, j \leq 4$.

\section{Proof of Theorem 1.1}

We need the following two lemmas proved by Fu and Hong in $[\mathrm{FH}]$.

Lemma 4.1. ([FH])

$$
\begin{gathered}
\omega_{23}^{4}\left(\lambda_{3}-\lambda_{4}\right)=\omega_{32}^{4}\left(\lambda_{2}-\lambda_{4}\right)=\omega_{43}^{2}\left(\lambda_{3}-\lambda_{2}\right), \\
\omega_{23}^{4} \omega_{32}^{4}+\omega_{34}^{2} \omega_{43}^{2}+\omega_{24}^{3} \omega_{42}^{3}=0,
\end{gathered}
$$




$$
\omega_{23}^{4}\left(\omega_{33}^{1}-\omega_{44}^{1}\right)=\omega_{32}^{4}\left(\omega_{22}^{1}-\omega_{44}^{1}\right)=\omega_{43}^{2}\left(\omega_{33}^{1}-\omega_{22}^{1}\right) .
$$

Lemma 4.2. ([FH])

$$
\begin{aligned}
& \omega_{22}^{1} \omega_{33}^{1}-2 \omega_{23}^{4} \omega_{32}^{4}=-\lambda_{2} \lambda_{3}-c, \\
& \omega_{22}^{1} \omega_{44}^{1}-2 \omega_{24}^{3} \omega_{42}^{3}=-\lambda_{2} \lambda_{4}-c, \\
& \omega_{33}^{1} \omega_{44}^{1}-2 \omega_{34}^{2} \omega_{43}^{2}=-\lambda_{3} \lambda_{4}-c .
\end{aligned}
$$

\section{The proof of Theorem 1.1:}

Let $M^{4}$ be a biharmonic hypersurface in $\mathbb{R}^{5}(c)(c \neq 0)$. There exists a smooth function $a$ such that

$$
\omega_{23}^{4}=a\left(\lambda_{2}-\lambda_{3}\right)\left(\lambda_{2}-\lambda_{4}\right) .
$$

From (4.1) and (4.7) we have

$$
\begin{aligned}
& \omega_{34}^{2}=a\left(\lambda_{3}-\lambda_{4}\right)\left(\lambda_{3}-\lambda_{2}\right), \\
& \omega_{42}^{3}=a\left(\lambda_{4}-\lambda_{2}\right)\left(\lambda_{4}-\lambda_{3}\right) .
\end{aligned}
$$

Taking $X=e_{1}, Y=e_{2}, Z=e_{3}$ in Gauss equation (2.4), we have (4.10)

$$
\begin{aligned}
e_{1}(a)= & -\frac{a}{3\left(\lambda_{2}-\lambda_{3}\right)\left(\lambda_{2}-\lambda_{4}\right)}\left\{\left(5 \lambda_{2}^{2}+\lambda_{2} \lambda_{3}-\lambda_{3}^{2}+\lambda_{2} \lambda_{4}-5 \lambda_{3} \lambda_{4}-\lambda_{4}^{2}\right) \omega_{22}^{1}\right. \\
& +\left(-\lambda_{2}^{2}-4 \lambda_{2} \lambda_{3}+4 \lambda_{3} \lambda_{4}+\lambda_{4}^{2}\right) \omega_{33}^{1} \\
& \left.+\left(-\lambda_{2}^{2}+\lambda_{3}^{2}-4 \lambda_{2} \lambda_{4}+4 \lambda_{3} \lambda_{4}\right) \omega_{44}^{1}\right\} .
\end{aligned}
$$

Taking $X=e_{1}, Y=e_{3}, Z=e_{2}$ in Gauss equation (2.4), we have

$$
\begin{aligned}
e_{1}(a)= & -\frac{a}{3\left(\lambda_{2}-\lambda_{3}\right)\left(\lambda_{3}-\lambda_{4}\right)}\left\{\left(4 \lambda_{2} \lambda_{3}+\lambda_{3}^{2}-4 \lambda_{2} \lambda_{4}-\lambda_{4}^{2}\right) \omega_{22}^{1}\right. \\
& +\left(\lambda_{2}^{2}-\lambda_{2} \lambda_{3}-5 \lambda_{3}^{2}+5 \lambda_{2} \lambda_{4}-\lambda_{3} \lambda_{4}+\lambda_{4}^{2}\right) \omega_{33}^{1} \\
& \left.+\left(-\lambda_{2}^{2}+\lambda_{3}^{2}-4 \lambda_{2} \lambda_{4}+4 \lambda_{3} \lambda_{4}\right) \omega_{44}^{1}\right\} .
\end{aligned}
$$

Taking $X=e_{1}, Y=e_{4}, Z=e_{2}$ in Gauss equation (2.4), we have

$$
\begin{aligned}
e_{1}(a)= & -\frac{a}{3\left(\lambda_{2}-\lambda_{4}\right)\left(\lambda_{3}-\lambda_{4}\right)}\left\{\left(4 \lambda_{2} \lambda_{3}+\lambda_{3}^{2}-4 \lambda_{2} \lambda_{4}-\lambda_{4}^{2}\right) \omega_{22}^{1}\right. \\
& +\left(\lambda_{2}^{2}+4 \lambda_{2} \lambda_{3}-4 \lambda_{3} \lambda_{4}-\lambda_{4}^{2}\right) \omega_{33}^{1} \\
& \left.+\left(-\lambda_{2}^{2}-5 \lambda_{2} \lambda_{3}-\lambda_{3}^{2}+\lambda_{2} \lambda_{4}+\lambda_{3} \lambda_{4}+5 \lambda_{4}^{2}\right) \omega_{44}^{1}\right\} .
\end{aligned}
$$

$(4.10)+(4.11)+(4.12)$ implies

$$
e_{1}(a)=\frac{a}{9\left(\lambda_{2}-\lambda_{3}\right)\left(\lambda_{2}-\lambda_{4}\right)\left(\lambda_{3}-\lambda_{4}\right)}\left(k_{2} \omega_{22}^{1}-k_{3} \omega_{33}^{1}+k_{4} \omega_{44}^{1}\right),
$$

where

$$
\begin{aligned}
& k_{2}=\left(\lambda_{3}-\lambda_{4}\right)\left(-13 \lambda_{2}^{2}+2 \lambda_{3}^{2}+7 \lambda_{3} \lambda_{4}+2 \lambda_{4}^{2}+\lambda_{2} \lambda_{3}+\lambda_{2} \lambda_{4}\right), \\
& k_{3}=\left(\lambda_{2}-\lambda_{4}\right)\left(2 \lambda_{2}^{2}-13 \lambda_{3}^{2}+\lambda_{3} \lambda_{4}+2 \lambda_{4}^{2}+\lambda_{2} \lambda_{3}+7 \lambda_{2} \lambda_{4}\right), \\
& k_{4}=\left(\lambda_{2}-\lambda_{3}\right)\left(2 \lambda_{2}^{2}+7 \lambda_{2} \lambda_{3}+2 \lambda_{3}^{2}+\lambda_{2} \lambda_{4}+\lambda_{3} \lambda_{4}-13 \lambda_{4}^{2}\right) .
\end{aligned}
$$


Taking $X=e_{2}, Y=e_{4}, Z=e_{1}$ in Gauss equation (2.4), we have

$$
a\left\{\left(\lambda_{3}-\lambda_{4}\right) \omega_{22}^{1}-\left(\lambda_{2}-\lambda_{4}\right) \omega_{33}^{1}+\left(\lambda_{2}-\lambda_{3}\right) \omega_{44}^{1}\right\}=0 .
$$

We can rewrite the biharmonic equation (2.7) as

$$
-e_{1} e_{1}\left(\lambda_{1}\right)+e_{1}\left(\lambda_{1}\right)\left(\omega_{22}^{1}+\omega_{33}^{1}+\omega_{44}^{1}\right)+\lambda_{1}\left(8 c+4 \lambda_{1}^{2}-R\right)=0 .
$$

Taking $X=e_{2}, Y=e_{4}, Z=e_{2}$ in Gauss equation (2.4), we have $e_{2}(a)=0$ and

$$
\omega_{22}^{1} \omega_{44}^{1}+2 a^{2}\left(\lambda_{2}-\lambda_{3}\right)\left(\lambda_{2}-\lambda_{4}\right)^{2}\left(\lambda_{3}-\lambda_{4}\right)+\lambda_{2} \lambda_{4}+c=0 .
$$

By symmetry, we have $e_{3}(a)=e_{4}(a)=0$ and

$$
\begin{aligned}
& \omega_{33}^{1} \omega_{44}^{1}-2 a^{2}\left(\lambda_{2}-\lambda_{3}\right)\left(\lambda_{2}-\lambda_{4}\right)\left(\lambda_{3}-\lambda_{4}\right)^{2}+\lambda_{3} \lambda_{4}+c=0, \\
& \omega_{22}^{1} \omega_{33}^{1}+\lambda_{2} \lambda_{3}-2 a^{2}\left(\lambda_{2}-\lambda_{3}\right)^{2}\left(\lambda_{2}-\lambda_{4}\right)\left(\lambda_{3}-\lambda_{4}\right)+c=0 .
\end{aligned}
$$

Here we introduce the new variables $y_{1}, y_{2}, y_{3}$ by

$$
\begin{gathered}
y_{1}=\lambda_{2}+\lambda_{3}+\lambda_{4}=-3 \lambda_{1}, \\
y_{2}=\lambda_{2} \lambda_{3}+\lambda_{2} \lambda_{4}+\lambda_{3} \lambda_{4}, \\
y_{3}=\lambda_{2} \lambda_{3} \lambda_{4} .
\end{gathered}
$$

Next we consider two cases.

Case A: $a \neq 0$. From (4.14) we have

$$
\left(\lambda_{3}-\lambda_{4}\right) \omega_{22}^{1}-\left(\lambda_{2}-\lambda_{4}\right) \omega_{33}^{1}+\left(\lambda_{2}-\lambda_{3}\right) \omega_{44}^{1}=0 .
$$

From (4.1) and (4.3), there exists smooth functions $\kappa$ and $\tau$ such that

$$
\omega_{i i}^{1}=\kappa \lambda_{i}+\tau, \quad i=2,3,4 .
$$

From (2.13) and (2.14), we have

$$
\begin{gathered}
e_{1}(\kappa)=-\frac{1}{3}\left(1+\kappa^{2}\right)\left(\lambda_{2}+\lambda_{3}+\lambda_{4}\right)+\kappa \tau, \\
e_{1}(\tau)=c-\frac{1}{3} \kappa \tau\left(\lambda_{2}+\lambda_{3}+\lambda_{4}\right)+\tau^{2} .
\end{gathered}
$$

$(4.16)+(4.17)+(4.18)$ implies

$$
3 c+\left(1+\kappa^{2}\right) y_{2}+2 \kappa \tau y_{1}+3 \tau^{2}=0 .
$$

From (4.26) we can solve for $y_{2}$

$$
y_{2}=-\frac{1}{1+\kappa^{2}}\left(3 c+2 \kappa \tau y_{1}+3 \tau^{2}\right)
$$

From (2.13), (2.14), (4.23), we have

$$
e_{1}\left(y_{1}\right)=\frac{4}{3} \kappa y_{1}^{2}-2 \kappa y_{2}+2 \tau y_{1} .
$$

From $(4.24),(4.25),(4.27),(4.28)$, we have

$$
\begin{aligned}
e_{1}\left(y_{1}^{2}-2 y_{2}\right) & =\frac{4}{3\left(1+\kappa^{2}\right)^{2}}\left(2 y_{1}^{3} \kappa\left(1+\kappa^{2}\right)^{2}+15 c y_{1}\left(\kappa+\kappa^{3}\right)+9 c\left(1+2 \kappa^{2}\right) \tau\right. \\
& \left.+y_{1}^{2}\left(1+\kappa^{2}\right)\left(2+13 \kappa^{2}\right) \tau+3 y_{1} \kappa\left(7+9 \kappa^{2}\right) \tau^{2}+9\left(1+2 \kappa^{2}\right) \tau^{3}\right) .
\end{aligned}
$$

From (2.13), (2.14), (4.23), we have

$$
e_{1}\left(\lambda_{2}^{2}+\lambda_{3}^{2}+\lambda_{4}^{2}\right)=\frac{2}{3}\left(4 y_{1}^{3} \kappa-11 y_{1} y_{2} \kappa+9 y_{3} \kappa+4 y_{1}^{2} \tau-6 y_{2} \tau\right) .
$$


From (4.29) and (4.30), we have

(4.31) $-c y_{1}-3 y_{3}-c y_{1} \kappa^{2}-6 y_{3} \kappa^{2}-3 y_{3} \kappa^{4}+6 c \kappa \tau-y_{1} \tau^{2}+3 y_{1} \kappa^{2} \tau^{2}+6 \kappa \tau^{3}=0$.

From (4.31), we can solve for $y_{3}$

$$
y_{3}=\frac{1}{3\left(1+\kappa^{2}\right)^{2}}\left(-c\left(1+\kappa^{2}\right) y_{1}+6 c \kappa \tau+\left(3 \kappa^{2}-1\right) \tau^{2} y_{1}+6 \kappa \tau^{3}\right) .
$$

Using (4.23), (4.15) can be rewritten as

(4.33) $27 c y_{1}-7 y_{1}^{3}+12 y_{1} y_{2}+8 y_{1}^{3} \kappa^{2}-27 y_{1} y_{2} \kappa^{2}+27 y_{3} \kappa^{2}+6 y_{1}^{2} \kappa \tau-18 y_{2} \kappa \tau=0$.

Differentiating (4.33) with respect to $e_{1}$, we have

$$
\begin{aligned}
& -684 c y_{1}^{2} \kappa-100 y_{1}^{4} \kappa+2106 c^{2} \kappa^{3}+324 c y_{1}^{2} \kappa^{3} \\
& -120 y_{1}^{4} \kappa^{3}+1008 c y_{1}^{2} \kappa^{5}+60 y_{1}^{4} \kappa^{5}+80 y_{1}^{4} \kappa^{7} \\
& -324 c y_{1} \tau-108 y_{1}^{3} \tau+648 c y_{1} \kappa^{2} \tau-618 y_{1}^{3} \kappa^{2} \tau \\
& +4806 c y_{1} \kappa^{4} \tau+330 y_{1}^{3} \kappa^{4} \tau+840 y_{1}^{3} \kappa^{6} \tau-1062 y_{1}^{2} \kappa \tau^{2} \\
& +5346 c \kappa^{3} \tau^{2}+558 y_{1}^{2} \kappa^{3} \tau^{2}+3240 y_{1}^{2} \kappa^{5} \tau^{2}-486 y_{1} \tau^{3} \\
& +324 y_{1} \kappa^{2} \tau^{3}+5400 y_{1} \kappa^{4} \tau^{3}+3240 \kappa^{3} \tau^{4}=0 .
\end{aligned}
$$

Differentiating (4.34) with respect to $e_{1}$, we have

$$
\begin{aligned}
& -972 c^{2} y_{1}+360 c y_{1}^{3}+100 y_{1}^{5}-29970 c^{2} y_{1} \kappa^{2} \\
& -14454 c y_{1}^{3} \kappa^{2}-1040 y_{1}^{5} \kappa^{2}+15390 c^{2} y_{1} \kappa^{4}-18684 c y_{1}^{3} \kappa^{4} \\
& -3000 y_{1}^{5} \kappa^{4}+44388 c^{2} y_{1} \kappa^{6}+7434 c y_{1}^{3} \kappa^{6}-1760 y_{1}^{5} \kappa^{6} \\
& +11304 c y_{1}^{3} \kappa^{8}+820 y_{1}^{5} \kappa^{8}+720 y_{1}^{5} \kappa^{10}-5832 c^{2} \kappa \tau \\
& -24732 c y_{1}^{2} \kappa \tau-2652 y_{1}^{4} \kappa \tau+62694 c^{2} \kappa^{3} \tau-77112 c y_{1}^{2} \kappa^{3} \tau \\
& -18294 y_{1}^{4} \kappa^{3} \tau+137538 c^{2} \kappa^{5} \tau+59022 c y_{1}^{2} \kappa^{5} \tau-16992 y_{1}^{4} \kappa^{5} \tau \\
& +111402 c y_{1}^{2} \kappa^{7} \tau+10290 y_{1}^{4} \kappa^{7} \tau+11640 y_{1}^{4} \kappa^{9} \tau-7290 c y_{1} \tau^{2} \\
& -1206 y_{1}^{3} \tau^{2}-77436 c y_{1} \kappa^{2} \tau^{2}-35964 y_{1}^{3} \kappa^{2} \tau^{2}+158922 c y_{1} \kappa^{4} \tau^{2} \\
& -58194 y_{1}^{3} \kappa^{4} \tau^{2}+333396 c y_{1} \kappa^{6} \tau^{2}+48564 y_{1}^{3} \kappa^{6} \tau^{2}+72000 y_{1}^{3} \kappa^{8} \tau^{2} \\
& -14580 c \kappa \tau^{3}-29268 y_{1}^{2} \kappa \tau^{3}+136566 c \kappa^{3} \tau^{3}-89154 y_{1}^{2} \kappa^{3} \tau^{3} \\
& +302778 c \kappa^{5} \tau^{3}+115074 y_{1}^{2} \kappa^{5} \tau^{3}+213840 y_{1}^{2} \kappa^{7} \tau^{3}-7290 y_{1} \tau^{4} \\
& -54270 y_{1} \kappa^{2} \tau^{4}+144180 y_{1} \kappa^{4} \tau^{4}+304560 y_{1} \kappa^{6} \tau^{4}-8748 \kappa \tau^{5} \\
& +73872 \kappa^{3} \tau^{5}+165240 \kappa^{5} \tau^{5}=0 .
\end{aligned}
$$

From (4.33) and (4.34), eliminating $\tau$ we have

$$
\sum_{m=0}^{8} P_{2 m} \kappa^{2 m}=0,
$$

where 


$$
\begin{aligned}
P_{0}= & -164025 c^{3} y_{1}^{4}-149445 c^{2} y_{1}^{6}-17739 c y_{1}^{8}-567 y_{1}^{10}, \\
P_{2}= & -157464 c^{4} y_{1}^{2}+988524 c^{3} y_{1}^{4}-1879848 c^{2} y_{1}^{6}+449388 c y_{1}^{8}-18072 y_{1}^{10}, \\
P_{4}= & 6114852 c^{4} y_{1}^{2}+8627715 c^{3} y_{1}^{4}-4223745 c^{2} y_{1}^{6}+79353 c y_{1}^{8}-8223 y_{1}^{10}, \\
P_{6}= & 6141096 c^{5}-46189440 c^{4} y_{1}^{2}+84187836 c^{3} y_{1}^{4}-36738684 c^{2} y_{1}^{6} \\
& +4146300 c y_{1}^{8}-172964 y_{1}^{10}, \\
P_{8}= & 6141096 c^{5}-223047756 c^{4} y_{1}^{2}+137387340 c^{3} y_{1}^{4}-83992140 c^{2} y_{1}^{6} \\
& +12535884 c y_{1}^{8}-488104 y_{1}^{10}, \\
P_{10}= & -35311302 c^{5}+54154494 c^{4} y_{1}^{2}-49487436 c^{3} y_{1}^{4}-69538500 c^{2} y_{1}^{6} \\
& +11334546 c y_{1}^{8}-413882 y_{1}^{10}, \\
P_{12}= & -18423288 c^{5}+1192107456 c^{4} y_{1}^{2}-355203792 c^{3} y_{1}^{4}-6360768 c^{2} y_{1}^{6} \\
& +3415752 c y_{1}^{8}-121088 y_{1}^{10}, \\
P_{14}= & 55269864 c^{5}+1533226968 c^{4} y_{1}^{2}-345382704 c^{3} y_{1}^{4}+18635184 c^{2} y_{1}^{6} \\
& +46152 c y_{1}^{8}-15496 y_{1}^{10}, \\
P_{16}= & 587865600 c^{4} y_{1}^{2}-105629184 c^{3} y_{1}^{4}+5505408 c^{2} y_{1}^{6}-36864 c y_{1}^{8}-2432 y_{1}^{10} .
\end{aligned}
$$

From (4.33) and (4.35), eliminating $\tau$ we have

$$
\sum_{m=0}^{13} Q_{2 m} \kappa^{2 m}=0
$$

where

$$
\begin{array}{rl}
Q_{0}= & 89813529 c^{4} y_{1}^{4}+623321244 c^{3} y_{1}^{6}+1108270998 c^{2} y_{1}^{8} \\
+ & 92935836 c y_{1}^{10}+1996569 y_{1}^{12}, \\
Q_{2}= & 7620155352 c^{5} y_{1}^{2}+18958390038 c^{4} y_{1}^{4}-2051878392 c^{3} y_{1}^{6} \\
+ & 18386833140 c^{2} y_{1}^{8}-3132184464 c y_{1}^{10}+199117734 y_{1}^{12} \\
Q_{4}= & 2550916800 c^{6}-3713237316 c^{5} y_{1}^{2}+396683144775 c^{4} y_{1}^{4} \\
- & 281478327804 c^{3} y_{1}^{6}+162873940914 c^{2} y_{1}^{8}-23218046136 c y_{1}^{10} \\
+ & 1170436927 y_{1}^{12}, \\
Q_{6}= & 21533989320 c^{6}-253030869900 c^{5} y_{1}^{2}+2983350761604 c^{4} y_{1}^{4} \\
& -2233514241576 c^{3} y_{1}^{6}+897578511552 c^{2} y_{1}^{8}-110846416140 c y_{1}^{10} \\
+ & 5032123316 y_{1}^{12}, \\
Q_{8}= & 169801776792 c^{6}-4722853102668 c^{5} y_{1}^{2}+19054644126723 c^{4} y_{1}^{4} \\
& -11292218972532 c^{3} y_{1}^{6}+3695744541258 c^{2} y_{1}^{8}-455003760600 c y_{1}^{10} \\
+ & 19351326643 y_{1}^{12}, \\
Q_{10} & 554978521890 c^{6}-30549126042468 c^{5} y_{1}^{2}+83834398712052 c^{4} y_{1}^{4} \\
& -42196006098576 c^{3} y_{1}^{6}+11288209375170 c^{2} y_{1}^{8}-1281849786108 c y_{1}^{10} \\
& +48941467416 y_{1}^{12},
\end{array}
$$




$$
\begin{aligned}
Q_{12}= & -310363669764 c^{6}-57933464951484 c^{5} y_{1}^{2}+211026987943857 c^{4} y_{1}^{4} \\
& -105270974689716 c^{3} y_{1}^{6}+23637191018346 c^{2} y_{1}^{8}-2258485653816 c y_{1}^{10} \\
& +72974080657 y_{1}^{12}, \\
Q_{14}= & -4681635955884 c^{6}+135848247734196 c^{5} y_{1}^{2}+275115121235820 c^{4} y_{1}^{4} \\
& -165192051148632 c^{3} y_{1}^{6}+32239821679308 c^{2} y_{1}^{8}-2470452821820 c y_{1}^{10} \\
& +62750560596 y_{1}^{12}, \\
Q_{16}= & -5901463112004 c^{6}+844079357467764 c^{5} y_{1}^{2}+106282529065260 c^{4} y_{1}^{4} \\
& -157860516846024 c^{3} y_{1}^{6}+27762314926644 c^{2} y_{1}^{8}-1657847338092 c y_{1}^{10} \\
& +29261950884 y_{1}^{12}, \\
Q_{18}= & 6145346701314 c^{6}+1705659405062004 c^{5} y_{1}^{2}-177158182581522 c^{4} y_{1}^{4} \\
& -87108572161368 c^{3} y_{1}^{6}+14676006828366 c^{2} y_{1}^{8}-656114701212 c y_{1}^{10} \\
& +5566963010 y_{1}^{12}, \\
Q_{20}= & 17073860098680 c^{6}+1765021213832760 c^{5} y_{1}^{2}-262032349848480 c^{4} y_{1}^{4} \\
& -25663081979952 c^{3} y_{1}^{6}+4778135352216 c^{2} y_{1}^{8}-147573429768 c y_{1}^{10} \\
& -751835760 y_{1}^{12}, \\
Q_{22}= & 9338536521864 c^{6}+941182952949648 c^{5} y_{1}^{2}-130448854754568 c^{4} y_{1}^{4} \\
& -5532982699104 c^{3} y_{1}^{6}+1153495908792 c^{2} y_{1}^{8}-23152890672 c y_{1}^{10} \\
& -611376440 y_{1}^{12}, \\
Q_{24}= & 205652969940096 c^{5} y_{1}^{2}-16668899694720 c^{4} y_{1}^{4}-2570855428224 c^{3} y_{1}^{6} \\
& +271365697152 c^{2} y_{1}^{8}-3749437440 c y_{1}^{10}-144665088 y_{1}^{12} \\
Q_{26}= & 3762339840000 c^{4} y_{1}^{4}-676026777600 c^{3} y_{1}^{6}+35234611200 c^{2} y_{1}^{8} \\
& -235929600 c y_{1}^{10}-15564800 y_{1}^{12} . \\
&
\end{aligned}
$$

From (4.36), (4.37), eliminating $\kappa$ we get a polynomial of $y_{1}$ with constant coefficients of degree 428 and then $y_{1}$ is a constant, which is a contradiction.

Case B: $a=0$. (4.4)-(4.6) becomes

$$
\begin{aligned}
& \omega_{22}^{1} \omega_{33}^{1}=-\lambda_{2} \lambda_{3}-c, \\
& \omega_{22}^{1} \omega_{44}^{1}=-\lambda_{2} \lambda_{4}-c, \\
& \omega_{33}^{1} \omega_{44}^{1}=-\lambda_{3} \lambda_{4}-c .
\end{aligned}
$$

From the assumption $c \neq 0$ and (4.38)-(4.40), we can get $\omega_{i i}^{1} \neq 0$ and $\lambda_{i} \lambda_{j}+c \neq 0$ for $2 \leq i, j \leq 4, i \neq j$, then

$$
\begin{aligned}
& \omega_{33}^{1}=-\frac{\lambda_{2} \lambda_{3}+c}{\omega_{22}^{1}}, \\
& \omega_{44}^{1}=-\frac{\lambda_{2} \lambda_{4}+c}{\omega_{22}^{1}},
\end{aligned}
$$




$$
\left(\omega_{22}^{1}\right)^{2}=-\frac{\left(\lambda_{2} \lambda_{3}+c\right)\left(\lambda_{2} \lambda_{4}+c\right)}{\lambda_{3} \lambda_{4}+c} .
$$

From (2.13), (2.14), (4.41)-(4.43), we have

$$
e_{1}\left(y_{1}\right)=-\frac{1}{3 \omega_{22}^{1}\left(\lambda_{3} \lambda_{4}+c\right)}\left(6 c^{2} y_{1}+5 c y_{1} y_{2}-9 c y_{3}+4 y_{1}^{2} y_{3}-6 y_{2} y_{3}\right),
$$

$e_{1}\left(y_{2}\right)=-\frac{1}{3 \omega_{22}^{1}\left(\lambda_{3} \lambda_{4}+c\right)}\left(2 c^{2} y_{1}^{2}+6 c^{2} y_{2}+c y_{1}^{2} y_{2}+6 c y_{2}^{2}-3 c y_{1} y_{3}+5 y_{1} y_{2} y_{3}-9 y_{3}^{2}\right)$,

$$
e_{1}\left(y_{3}\right)=-\frac{1}{3 \omega_{22}^{1}\left(\lambda_{3} \lambda_{4}+c\right)}\left(c^{2} y_{1} y_{2}+9 c^{2} y_{3}+2 c y_{1}^{2} y_{3}+6 c y_{2} y_{3}+6 y_{1} y_{3}^{2}\right) .
$$

Using (4.41)-(4.43), (4.15) can be rewritten as

$$
\begin{aligned}
& 27 c^{4} y_{1}-7 c^{3} y_{1}^{3}+36 c^{3} y_{1} y_{2}-7 c^{2} y_{1}^{3} y_{2}+7 c^{2} y_{1} y_{2}^{2}+27 c^{3} y_{3} \\
+ & 27 c^{2} y_{1}^{2} y_{3}-7 c y_{1}^{4} y_{3}+45 c^{2} y_{2} y_{3}+c y_{1}^{2} y_{2} y_{3}+24 c y_{2}^{2} y_{3}+54 c y_{1} y_{3}^{2} \\
- & 15 y_{1}^{3} y_{3}^{2}+39 y_{1} y_{2} y_{3}^{2}-27 y_{3}^{3}=0 .
\end{aligned}
$$

Differentiating (4.47) with respect to $e_{1}$, we have

$$
\begin{aligned}
& 162 c^{6} y_{1}-54 c^{5} y_{1}^{3}-14 c^{4} y_{1}^{5}+594 c^{5} y_{1} y_{2}-182 c^{4} y_{1}^{3} y_{2}-14 c^{3} y_{1}^{5} y_{2} \\
+ & 567 c^{4} y_{1} y_{2}^{2}-132 c^{3} y_{1}^{3} y_{2}^{2}+143 c^{3} y_{1} y_{2}^{3}+900 c^{4} y_{1}^{2} y_{3}-238 c^{3} y_{1}^{4} y_{3}-14 c^{2} y_{1}^{6} y_{3} \\
+ & 351 c^{4} y_{2} y_{3}+1395 c^{3} y_{1}^{2} y_{2} y_{3}-328 c^{2} y_{1}^{4} y_{2} y_{3}+765 c^{3} y_{2}^{2} y_{3}+420 c^{2} y_{1}^{2} y_{2}^{2} y_{3}+390 c^{2} y_{2}^{3} y_{3} \\
+ & 513 c^{3} y_{1} y_{3}^{2}+444 c^{2} y_{1}^{3} y_{3}^{2}-214 c y_{1}^{5} y_{3}^{2}+1890 c^{2} y_{1} y_{2} y_{3}^{2}-23 c y_{1}^{3} y_{2} y_{3}^{2}+1269 c y_{1} y_{2}^{2} y_{3}^{2} \\
- & 1620 c^{2} y_{3}^{3}+981 c y_{1}^{2} y_{3}^{3}-360 y_{1}^{4} y_{3}^{3}-1593 c y_{2} y_{3}^{3}+1089 y_{1}^{2} y_{2} y_{3}^{3}-234 y_{2}^{2} y_{3}^{3}-837 y_{1} y_{3}^{4}=0 .
\end{aligned}
$$

Differentiating (4.48) with respect to $e_{1}$, we have

$$
\begin{aligned}
& 972 c^{8} y_{1}+216 c^{7} y_{1}^{3}-784 c^{6} y_{1}^{5}-28 c^{5} y_{1}^{7} \\
+ & 7938 c^{7} y_{1} y_{2}-1416 c^{6} y_{1}^{3} y_{2}-1802 c^{5} y_{1}^{5} y_{2}-28 c^{4} y_{1}^{7} y_{2} \\
+ & 17091 c^{6} y_{1} y_{2}^{2}-4395 c^{5} y_{1}^{3} y_{2}^{2}-1026 c^{4} y_{1}^{5} y_{2}^{2}+13836 c^{5} y_{1} y_{2}^{3} \\
- & 2715 c^{4} y_{1}^{3} y_{2}^{3}+3679 c^{4} y_{1} y_{2}^{4}-1458 c^{7} y_{3}+19926 c^{6} y_{1}^{2} y_{3} \\
- & 2736 c^{5} y_{1}^{4} y_{3}-2000 c^{4} y_{1}^{6} y_{3}-28 c^{3} y_{1}^{8} y_{3}-1053 c^{6} y_{2} y_{3} \\
+ & 65034 c^{5} y_{1}^{2} y_{2} y_{3}-13479 c^{4} y_{1}^{4} y_{2} y_{3}-2266 c^{3} y_{1}^{6} y_{2} y_{3}+11610 c^{5} y_{2}^{2} y_{3} \\
+ & +67221 c^{4} y_{1}^{2} y_{2}^{2} y_{3}-11346 c^{3} y_{1}^{4} y_{2}^{2} y_{3}+19611 c^{4} y_{2}^{3} y_{3}+21341 c^{3} y_{1}^{2} y_{2}^{3} y_{3} \\
+ & 8502 c^{3} y_{2}^{4} y_{3}-10287 c^{5} y_{1} y_{3}^{2}+40437 c^{4} y_{1}^{3} y_{3}^{2}-11912 c^{3} y_{1}^{5} y_{3}^{2} \\
- & 1276 c^{2} y_{1}^{7} y_{3}^{2}+13716 c^{4} y_{1} y_{2} y_{3}^{2}+72372 c^{3} y_{1}^{3} y_{2} y_{3}^{2}-17465 c^{2} y_{1}^{5} y_{2} y_{3}^{2} \\
+ & 64944 c^{3} y_{1} y_{2}^{2} y_{3}^{2}+28074 c^{2} y_{1}^{3} y_{2}^{2} y_{3}^{2}+39249 c^{2} y_{1} y_{2}^{3} y_{3}^{2}-51516 c^{4} y_{3}^{3} \\
+ & 3348 c^{3} y_{1}^{2} y_{3}^{3}+13011 c^{2} y_{1}^{4} y_{3}^{3}-9008 c y_{1}^{6} y_{3}^{3}-11558 c^{3} y_{2} y_{3}^{3} \\
+ & +78183 c^{2} y_{1}^{2} y_{2} y_{3}^{3}-304 c y_{1}^{4} y_{2} y_{3}^{3}-80649 c^{2} y_{2}^{2} y_{3}^{3}+68562 c y_{1}^{2} y_{2}^{2} y_{3}^{3} \\
- & -14634 c y_{2}^{3} y_{3}^{3}-94203 c^{2} y_{1} y_{3}^{4}+28710 c y_{1}^{3} y_{3}^{4}-12240 y_{1}^{5} y_{3}^{4} \\
- & 113724 c y_{1} y_{2} y_{3}^{4}+42399 y_{1}^{3} y_{2} y_{3}^{4}-19620 y_{1} y_{2}^{2} y_{3}^{4}+21870 c y_{3}^{5} \\
- & 33237 y_{1}^{2} y_{3}^{5}+9234 y_{2} y_{3}^{5}=0 .
\end{aligned}
$$


From(4.47), (4.48), eliminating $y_{2}$ we have

$$
\begin{aligned}
& \left(c y_{1}-3 y_{3}\right)\left(c^{3}-c y_{1} y_{3}-2 y_{3}^{2}\right)\left(961551 c^{7} y_{1}^{4}-550638 c^{6} y_{1}^{6}\right. \\
+ & 92295 c^{5} y_{1}^{8}+1078 c^{4} y_{1}^{10}+3241134 c^{6} y_{1}^{3} y_{3} \\
- & 3718062 c^{5} y_{1}^{5} y_{3}+852336 c^{4} y_{1}^{7} y_{3}-16548 c^{3} y_{1}^{9} y_{3} \\
+ & 2071089 c^{5} y_{1}^{2} y_{3}^{2}-10454265 c^{4} y_{1}^{4} y_{3}^{2}+4078449 c^{3} y_{1}^{6} y_{3}^{2} \\
- & 228795 c^{2} y_{1}^{8} y_{3}^{2}-4234032 c^{4} y_{1} y_{3}^{3}-9157698 c^{3} y_{1}^{3} y_{3}^{3} \\
+ & 9454374 c^{2} y_{1}^{5} y_{3}^{3}-621684 c y_{1}^{7} y_{3}^{3}-4185918 c^{3} y_{3}^{4} \\
+ & 4435965 c^{2} y_{1}^{2} y_{3}^{4}+10034766 c y_{1}^{4} y_{3}^{4}-479115 y_{1}^{6} y_{3}^{4} \\
+ & \left.7050888 c y_{1} y_{3}^{5}+4106700 y_{1}^{3} y_{3}^{5}+2217618 y_{3}^{6}\right)=0 .
\end{aligned}
$$

From(4.47), (4.49), eliminating $y_{2}$ we have

$$
\begin{aligned}
&\left(c y_{1}-3 y_{3}\right)\left(c^{3}-c y_{1} y_{3}-2 y_{3}^{2}\right)\left(121234158 c^{12} y_{1}^{5}+4502127582 c^{11} y_{1}^{7}\right. \\
&- 4130694792 c^{10} y_{1}^{9}+1312681986 c^{9} y_{1}^{11}-138793970 c^{8} y_{1}^{13} \\
&--1131900 c^{7} y_{1}^{15}-6493539798 c^{11} y_{1}^{4} y_{3}+17508226059 c^{10} y_{1}^{6} y_{3} \\
&- 26579271393 c^{9} y_{1}^{8} y_{3}+12168575541 c^{8} y_{1}^{10} y_{3}-1901077395 c^{7} y_{1}^{12} y_{3} \\
&+ 41618150 c^{6} y_{1}^{14} y_{3}-19265759766 c^{10} y_{1}^{3} y_{3}^{2}+4495598658 c^{9} y_{1}^{5} y_{3}^{2} \\
&- 42049903410 c^{8} y_{1}^{7} y_{3}^{2}+42284538216 c^{7} y_{1}^{9} y_{3}^{2}-10550910060 c^{6} y_{1}^{11} y_{3}^{2} \\
&++560212114 c^{5} y_{1}^{13} y_{3}^{2}-6970891914 c^{9} y_{1}^{2} y_{3}^{3}-13811443002 c^{8} y_{1}^{4} y_{3}^{3} \\
&+ 83680475085 c^{7} y_{1}^{6} y_{3}^{3}+21298486113 c^{6} y_{1}^{8} y_{3}^{3}-21213196659 c^{5} y_{1}^{10} y_{3}^{3} \\
&+ 1830863937 c^{4} y_{1}^{12} y_{3}^{3}+30101526828 c^{8} y_{1} y_{3}^{4}+45869158224 c^{7} y_{1}^{3} y_{3}^{4} \\
&+ 279229797288 c^{6} y_{1}^{5} y_{3}^{4}-232982629074 c^{5} y_{1}^{7} y_{3}^{4}+23242316040 c^{4} y_{1}^{9} y_{3}^{4} \\
&- 602724402 c^{3} y_{1}^{11} y_{3}^{4}+23531498892 c^{7} y_{3}^{5}+95793204717 c^{6} y_{1}^{2} y_{3}^{5} \\
&- 35556908661 c^{5} y_{1}^{4} y_{3}^{5}-505869269463 c^{4} y_{1}^{6} y_{3}^{5}+173198646333 c^{3} y_{1}^{8} y_{3}^{5} \\
&--12359609514 c^{2} y_{1}^{10} y_{3}^{5}-28067406876 c^{5} y_{1} y_{3}^{6}-471693791502 c^{4} y_{1}^{3} y_{3}^{6} \\
&- 188063792496 c^{3} y_{1}^{5} y_{3}^{6}+305129824146 c^{2} y_{1}^{7} y_{3}^{6}-20136670848 c y_{1}^{9} y_{3}^{6} \\
&--99047493522 c^{4} y_{3}^{7}-128261179269 c^{3} y_{1}^{2} y_{3}^{7}+309915643131 c^{2} y_{1}^{4} y_{3}^{7} \\
&+ 254417953509 c y_{1}^{6} y_{3}^{7}-9828590625 y_{1}^{8} y_{3}^{7}+92615758344 c^{2} y_{1} y_{3}^{8} \\
&+ 252963396576 c y_{1}^{3} y_{3}^{8}+90957709080 y_{1}^{5} y_{3}^{8} \\
&+\left.45154416006 c y_{3}^{9}+25402919166 y_{1}^{2} y_{3}^{9}\right)=0 . \\
&
\end{aligned}
$$

Next we check three subcases.

Case B.1: $c y_{1}-3 y_{3}=0$. Substituting $y_{3}=\frac{c y_{1}}{3}$ into (4.47), (4.48) gives

$$
\left(9 c+4 y_{1}^{2}+9 y_{2}\right)\left(12 c-3 y_{1}^{2}+5 y_{2}\right)=0,
$$

$$
\left(9 c+4 y_{1}^{2}+9 y_{2}\right)\left(162 c^{2}+171 c y_{1}^{2}-94 y_{1}^{4}+549 c y_{2}+19 y_{1}^{2} y_{2}+273 y_{2}^{2}\right)=0 .
$$

From (4.52), (4.53), we consider the two following subcases:

(i) $9 c+4 y_{1}^{2}+9 y_{2} \neq 0$, or

(ii) $9 c+4 y_{1}^{2}+9 y_{2}=0$. 
If (i) holds, by eliminating $y_{2}$ we can get that $y_{1}$ satisfies a polynomial with constant coefficients and $y_{1}$ is a constant. If (ii) holds, taking $9 c+4 y_{1}^{2}+9 y_{2}=0$ into (4.44) we get $e_{1}\left(y_{1}\right)=0$ and $y_{1}$ is also a constant. So in both subcases we get that $y_{1}$ is a constant, which is a contradiction.

Case B.2: $c^{3}-c y_{1} y_{3}-2 y_{3}^{2}=0$. Since $c \neq 0$, we solve for $y_{1}$ in terms of $y_{3}$. Substituting $y_{1}=\frac{c^{3}-2 y_{3}^{2}}{c y_{3}}$ into (4.47) and (4.48), we have

$$
\begin{aligned}
\left(2 c^{3}+\right. & \left.c^{2} y_{2}-y_{3}^{2}\right)\left(7 c^{9}-65 c^{6} y_{3}^{2}-7 c^{5} y_{2} y_{3}^{2}+89 c^{3} y_{3}^{4}-10 c^{2} y_{2} y_{3}^{4}+8 y_{3}^{6}\right)=0 \\
& \left(2 c^{3}+c^{2} y_{2}-y_{3}^{2}\right)\left(14 c^{15}+106 c^{12} y_{3}^{2}+132 c^{11} y_{2} y_{3}^{2}-2004 c^{9} y_{3}^{4}\right. \\
- & 1493 c^{8} y_{2} y_{3}^{4}-143 c^{7} y_{2}^{2} y_{3}^{4}+5885 c^{6} y_{3}^{6}+2429 c^{5} y_{2} y_{3}^{6} \\
- & \left.104 c^{4} y_{2}^{2} y_{3}^{6}-3842 c^{3} y_{3}^{8}-68 c^{2} y_{2} y_{3}^{8}+192 y_{3}^{10}\right)=0
\end{aligned}
$$

From (4.54), (4.55), we consider the two following subcases:

(i) $2 c^{3}+c^{2} y_{2}-y_{3}^{2} \neq 0$, or

(ii) $2 c^{3}+c^{2} y_{2}-y_{3}^{2}=0$.

If (i) holds, by eliminating $y_{2}$ we can get that $y_{3}$ satisfies a polynomial with constant coefficients and $y_{3}$ is a constant. If (ii) holds, taking $2 c^{3}+c^{2} y_{2}-y_{3}^{2}=0$ into (4.46) we get $e_{1}\left(y_{3}\right)=0$ and $y_{3}$ is also a constant. So in both subcases we get that $y_{3}$ is a constant, then $y_{1}$ is a constant, which is a contradiction.

Case B.3: $\left(c y_{1}-3 y_{3}\right)\left(c^{3}-c y_{1} y_{3}-2 y_{3}^{2}\right) \neq 0$. From (4.50), (4.51), we have

$$
\begin{aligned}
& 961551 c^{7} y_{1}^{4}-550638 c^{6} y_{1}^{6}+92295 c^{5} y_{1}^{8}+1078 c^{4} y_{1}^{10} \\
+ & 3241134 c^{6} y_{1}^{3} y_{3}-3718062 c^{5} y_{1}^{5} y_{3}+852336 c^{4} y_{1}^{7} y_{3}-16548 c^{3} y_{1}^{9} y_{3} \\
+ & 2071089 c^{5} y_{1}^{2} y_{3}^{2}-10454265 c^{4} y_{1}^{4} y_{3}^{2}+4078449 c^{3} y_{1}^{6} y_{3}^{2}-228795 c^{2} y_{1}^{8} y_{3}^{2} \\
- & 4234032 c^{4} y_{1} y_{3}^{3}-9157698 c^{3} y_{1}^{3} y_{3}^{3}+9454374 c^{2} y_{1}^{5} y_{3}^{3}-621684 c y_{1}^{7} y_{3}^{3} \\
- & 4185918 c^{3} y_{3}^{4}+4435965 c^{2} y_{1}^{2} y_{3}^{4}+10034766 c y_{1}^{4} y_{3}^{4}-479115 y_{1}^{6} y_{3}^{4} \\
+ & 7050888 c y_{1} y_{3}^{5}+4106700 y_{1}^{3} y_{3}^{5}+2217618 y_{3}^{6}=0, \\
& 121234158 c^{12} y_{1}^{5}+4502127582 c^{11} y_{1}^{7}-4130694792 c^{10} y_{1}^{9} \\
+ & 1312681986 c^{9} y_{1}^{11}-138793970 c^{8} y_{1}^{13}-1131900 c^{7} y_{1}^{15} \\
- & 6493539798 c^{11} y_{1}^{4} y_{3}+17508226059 c^{10} y_{1}^{6} y_{3}-26579271393 c^{9} y_{1}^{8} y_{3} \\
+ & 12168575541 c^{8} y_{1}^{10} y_{3}-1901077395 c^{7} y_{1}^{12} y_{3}+41618150 c^{6} y_{1}^{14} y_{3} \\
- & 19265759766 c^{10} y_{1}^{3} y_{3}^{2}+4495598658 c^{9} y_{1}^{5} y_{3}^{2}-42049903410 c^{8} y_{1}^{7} y_{3}^{2} \\
+ & 42284538216 c^{7} y_{1}^{9} y_{3}^{2}-10550910060 c^{6} y_{1}^{11} y_{3}^{2}+560212114 c^{5} y_{1}^{13} y_{3}^{2} \\
- & 6970891914 c^{9} y_{1}^{2} y_{3}^{3}-13811443002 c^{8} y_{1}^{4} y_{3}^{3}+83680475085 c^{7} y_{1}^{6} y_{3}^{3} \\
+ & 21298486113 c^{6} y_{1}^{8} y_{3}^{3}-21213196659 c^{5} y_{1}^{10} y_{3}^{3}+1830863937 c^{4} y_{1}^{12} y_{3}^{3} \\
+ & 30101526828 c^{8} y_{1} y_{3}^{4}+45869158224 c^{7} y_{1}^{3} y_{3}^{4}+279229797288 c^{6} y_{1}^{5} y_{3}^{4} \\
- & 232982629074 c^{5} y_{1}^{7} y_{3}^{4}+23242316040 c^{4} y_{1}^{9} y_{3}^{4}-602724402 c^{3} y_{1}^{11} y_{3}^{4} \\
+ & 23531498892 c^{7} y_{3}^{5}+95793204717 c^{6} y_{1}^{2} y_{3}^{5}-35556908661 c^{5} y_{1}^{4} y_{3}^{5} \\
- & 505869269463 c^{4} y_{1}^{6} y_{3}^{5}+173198646333 c^{3} y_{1}^{8} y_{3}^{5}-12359609514 c^{2} y_{1}^{10} y_{3}^{5}
\end{aligned}
$$




$$
\begin{aligned}
& -28067406876 c^{5} y_{1} y_{3}^{6}-471693791502 c^{4} y_{1}^{3} y_{3}^{6}-188063792496 c^{3} y_{1}^{5} y_{3}^{6} \\
& +305129824146 c^{2} y_{1}^{7} y_{3}^{6}-20136670848 c y_{1}^{9} y_{3}^{6}-99047493522 c^{4} y_{3}^{7} \\
& -128261179269 c^{3} y_{1}^{2} y_{3}^{7}+309915643131 c^{2} y_{1}^{4} y_{3}^{7}+254417953509 c y_{1}^{6} y_{3}^{7} \\
& -9828590625 y_{1}^{8} y_{3}^{7}+92615758344 c^{2} y_{1} y_{3}^{8}+252963396576 c y_{1}^{3} y_{3}^{8} \\
& +90957709080 y_{1}^{5} y_{3}^{8}+45154416006 c y_{3}^{9}+25402919166 y_{1}^{2} y_{3}^{9}=0 .
\end{aligned}
$$

From (4.56) and (4.57), eliminating $y_{3}$ we get a polynomial of $y_{1}$ with constant coefficients of degree 118 and then $y_{1}$ is a constant, that is, $\lambda_{1}$ is constant, which is a contradiction. Therefore we complete the proof of Theorem 1.1.

\section{REFERENCES}

[BMO1] A. Balmus, S. Montaldo and C. Oniciuc, Classification results for biharmonic submanifolds in spheres, Israel J. Math. 168 (2008), 201-220.

[BMO2] A. Balmus, S. Montaldo and C. Oniciuc, Biharmonic hypersurfaces in 4-dimensional space forms, Math. Nachr. 283 (2010), no. 12, 1696-1705.

[CMO1] R. Caddeo, S. Montaldo and C. Oniciuc, Biharmonic submanifolds of $\mathbb{S}^{3}$, Internat. J. Math. 12 (2001), no. 8, 867-876.

[CMO2] R. Caddeo, S. Montaldo and C. Oniciuc, Biharmonic submanifolds in spheres, Israel J. Math. 130 (2002), 109-123.

[C1] B. -Y. Chen, Some open problems and conjectures on submanifolds of finite type, Soochow J. Math. 17 (1991) no. 2, 169-188.

[C2] B. -Y. Chen, Some open problems and conjectures on submanifolds of finite type: recent development, Tamkang J. Math. 45:1 (2014), 87-108.

[C3] B. -Y. Chen, Total Mean curvature and submanifolds of finite type, 2nd Edition, World Scientific, Hackensack, NJ, 2015.

[CM] B. -Y. Chen and M. I. Munteanu, Biharmonic ideal hypersurfaces in Euclidean spaces, Differ- ential Geom. Appl. 31 (2013), 1-16.

[ES] J. Eells and J. H. Sampson, Harmonic mappings of Riemannian manifolds, Amer. J. Math. 86 (1964), 109-160.

[F1] Y. Fu, Biharmonic hypersurfaces with three distinct principal curvatures in Euclidean space, Tohoku Math. J. 67 (2015), 465-479.

[F2] Y. Fu, Biharmonic hypersurfaces with three distinct principal curvatures in spheres, Math. Nachr. 288 (2015) 7, 763-774.

[F3] Y. Fu, On biharmonic hypersurfaces with constant scalar curvature in $\mathbb{S}^{5}$, Proc. Amer. Math. Soc. 143 (2015), 5399-5409.

[FH] Y. Fu and M. C. Hong, Biharmonic hypersurfaces with constant scalar curvature in space forms, Pacific J. Math. 294 (2) (2018), 329-350.

[FHZ] Y. Fu, M. C. Hong and X. Zhan, On Chen's biharmonic conjecture for hypersurfaces in $\mathbb{R}^{5}$, arXiv: 2006.07612v2.

$[\mathrm{HV}] \mathrm{T}$. Hasanis and T. Vlachos, Hypersurfaces in $\mathbb{E}^{4}$ with harmonic mean curvature vector field, Math. Nachr. 172 (1995), 145-169.

[J1] G. Y. Jiang, 2-Harmonic maps and their first and second variational formulas, Chin. Ann. Math. Ser. A 7 (1986), 389-402.

[J2] G. Y. Jiang, Some non-existence theorems of 2-harmonic isometric immersions into Euclidean spaces, Chin. Ann. Math. Ser. A 8 (1987), 376-383.

[OT] Y.-L. Ou and L. Tang, On the generalized Chen's conjecture on biharmonic submanifolds, Michigan Math. J. 61 (2012), 531-542.

[OC] Y.-L. Ou and B. -Y. Chen, Biharmonic submanifolds and biharmonic maps in Riemannian geometry, World Scientific Publishing, Hackensack, NJ, 2020. 
Department of Mathematical Sciences, Tsinghua University, Beijing 100084, People's Republic of China

E-mail address: gzd15@mails.tsinghua.edu.cn

Department of Mathematical Sciences, Tsinghua University, Beijing 100084, People's Republic of China

E-mail address: lihz@tsinghua.edu.cn

LMi-Laboratoire de Mathématiques pour l'Ingénieur, Université Polytechnique Hauts de France, 59313 Valenciennes, France, and KU Leuven, Department of Mathematics, Leuven, Belgium

E-mail address: luc.vrancken@uphf.fr 